\title{
A cryogenic magneto-optical device for long wavelength radiation
}

Cite as: Rev. Sci. Instrum. 91, 075103 (2020); https://doi.org/10.1063/5.0011348

Submitted: 21 April 2020 . Accepted: 05 June 2020 . Published Online: 02 July 2020

(iD S. J. Rezvani, (D) D. Di Gioacchino, (D) S. Tofani, (D) A. D’Arco, (D) C. Ligi, S. Lupi, (D) C. Gatti, M. Cestelli Guidi, and (iD) A. Marcelli

\section{ARTICLES YOU MAY BE INTERESTED IN}

Closed-cycle, low-vibration $4 \mathrm{~K}$ cryostat for ion traps and other applications

Review of Scientific Instruments 90, 065104 (2019); https://doi.org/10.1063/1.5088593

A laser powder bed fusion system for in situ x-ray diffraction with high-energy synchrotron radiation

Review of Scientific Instruments 91, 075104 (2020); https://doi.org/10.1063/1.5143766

Comparative study for intermediate crystal size of $\mathrm{Nal}(\mathrm{TI})$ scintillation detector

Review of Scientific Instruments 91, 073105 (2020); https://doi.org/10.1063/5.0005243

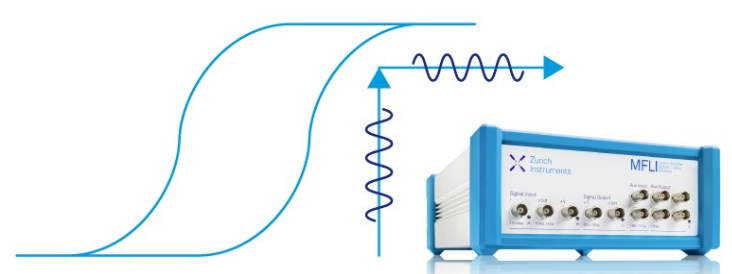

Webinar

How to Characterize Magnetic

Materials Using Lock-in Amplifiers

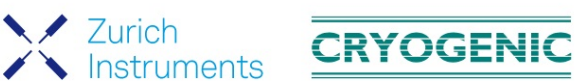




\title{
A cryogenic magneto-optical device for long wavelength radiation
}

\author{
Cite as: Rev. Sci. Instrum. 91, 075103 (2020); doi: 10.1063/5.0011348 \\ Submitted: 21 April 2020 - Accepted: 5 June 2020 • \\ Published Online: 2 July 2020
}

\author{
S. J. Rezvani, ${ }^{1,2, a)}$ (D) D. Di Gioacchino, ${ }^{1}$ (D) S. Tofani, ${ }^{3}$ (D) A. D'Arco, ${ }^{4,5}$ (D) C. Ligi, ${ }^{1}$ (D) S. Lupi, ${ }^{1,4}$ C. Gatti, \\ M. Cestelli Guidi,' and A. Marcelli ${ }^{1,6}$ \\ AFFILIATIONS \\ ${ }^{1}$ INFN - Laboratori Nazionali di Frascati, Via Enrico 54, 00044 Frascati (RM), Italy \\ ${ }^{2}$ Consiglio Nazionale delle Ricerche (CNR), CNR-IOM, 34149 Basovizza, Italy \\ ${ }^{3}$ Department of Information Engineering, Electronics and Telecommunications, "Sapienza" University of Rome, \\ 00184 Rome, Italy \\ ${ }^{4}$ Department of Physics, Sapienza University of Rome, 00185 Rome, Italy \\ ${ }^{5}$ INFN - Roma1, P.le Aldo Moro 2, 00185 Rome, Italy \\ ${ }^{6}$ RICMASS, Rome International Center for Materials Science Superstripes, Via dei Sabelli 119A, 00185 Rome, Italy
}

\begin{abstract}
a) Author to whom correspondence should be addressed: rezvani@lnf.infn.it
ABSTRACT

We present here a small-scale liquid helium immersion cryostat with an innovative optical setup suitable to work in long wavelength radiation ranges and under an applied magnetic field. The cryostat is a multi-stage device with several shielding in addition to several optical stages. The system has been designed with an external liquid nitrogen boiler to reduce liquid bubbling. The optical and mechanical properties of the optical elements were calculated and optimized for the designed configuration, while the optical layout has been simulated and optimized among different configurations based on the geometry of the device. The final design has been optimized for low-noise radiation measurements of proximity junction arrays under an applied magnetic field in the wavelength range $\lambda=250 \mu \mathrm{m}-2500 \mu \mathrm{m}$.
\end{abstract}

Published under license by AIP Publishing. https://doi.org/10.1063/5.0011348

\section{INTRODUCTION}

Cryogenic temperatures are required in many research studies such as superconductivity and superfluidity, surface and interface, advanced spectroscopies, and in the R\&D of low-noise detectors. As an example, superconducting radiation detectors integrated into surface Paul traps for scalable quantum information processing require operation at cryogenic temperatures. ${ }^{1-3}$ Optical spectroscopies at low temperature usually allow resolving narrow features and trap unstable intermediates. ${ }^{4}$ Recently, long wavelength radiations have come to attention being a particular region of the electromagnetic spectrum interesting for multi-disciplinary applications for both basic research studies and technologies. ${ }^{5-7}$ This e.m. domain $\lambda=250 \mu \mathrm{m}-2500 \mu \mathrm{m}$ (energy range $0.5 \mathrm{meV}-4 \mathrm{meV}$ ) allows the investigation of several fundamental physical phenomena, e.g., phonon and plasmon dynamics, elementary particle physics, and possibly also cold dark matter. ${ }^{8-12}$ Furthermore, with its high transmission through a wide range of non-conducting materials, long wavelength radiation and, in particular, terahertz $(\mathrm{THz})$ radiation, hold a significant potential in several applications.

In recent years, the investigation of semiconducting and superconducting behaviors of many materials and the modulation of the electronic properties of systems with either low dimensionality $^{13-16}$ or surface structural modifications ${ }^{17-21}$ have been performed. It has been demonstrated that superconducting proximity junction arrays can be designed to behave as long wavelength radiation detectors. $^{22,23}$ The superconducting dynamics of such devices, based on non-localized vortex dynamics, can be modulated via external magnetic and electric fields. ${ }^{24}$ The response of these devices in the radio frequency domain, well below the expected characteristic frequency of the proximity junction, can be tuned via non-equilibrium phenomena induced by external perturbations. It has also been speculated that such devices may represent a new pathway toward robust, low noise, broadband and with high sensitivity low energy detectors. However, the investigation of the dynamics in such devices requires a low-noise cryostat with a highly stable temperature control that can 
be operated under external magnetic and electric fields. These cryogenic systems should also be designed with suitable optical arrangements compatible with this energy range. In a conventional cold finger optical system, the sample is cooled through its mechanical link to the cooling element either by boiling liquid helium or using a mechanical refrigerator. This approach presents several problems. The first is that the sample is cooled relatively slowly, and often, we observe the formation of crystallites on the sample surface. In addition, reaching the required temperatures and its fine control under the applied electric and magnetic fields is not an easy task. Finally, the optimization of the optical arrangement to operate in this extreme photon energy range is complex.

Within the framework of the project TERA, ${ }^{25}$ we have developed a small-scale liquid helium immersion cryostat with an embedded optical setup, aimed at the detection of the photo-response of novel proximity junction arrays under an applied magnetic field. In the following, we will present and discuss the different cooling stages, the shields, and the optical layout. The latter has been simulated and optimized based on the different in-operando configurations. The optical transmission and the mechanical resistance of the optical components have been calculated, optimized, and characterized.

\section{CRYOSTAT AND OPTICAL SETUP DESCRIPTION}

A schematic view of the immersion cryostat is shown in Fig. 1. The cryostat is designed to be a table top small-scale cryostat for facile optical alignments. The section views of the cryostat [Fig. 1(b)] show the vacuum chamber (2) surrounding the liquid nitrogen tank (3) in the upper half and the polished copper radiation shield in the lower one (11). The shield has a hole in correspondence to the optical path. At the center is the liquid helium container (4) inside which the experimental apparatus (1) is hosted. The inner container has a $10 \mathrm{~cm}$ diameter, a value that sets the maximum size of both the magnetic coils and sample holder. The inner stainless steel tube is provided at the bottom with a window assembly mounted on a Conflat (CF) flange that holds a $29 \mathrm{~mm}$ diameter optical window set in front of the sample.

The copper cooling jacket extends down the sides as well as below the window assembly to improve cooling and to speed up the initial cooling process. The external container is equipped with four vacuum-tight optical windows. All inner metal-metal connections, except the copper shield and the cryostat cover part that is connected by bolts, have been connected either by soldering or brazing. Inside upper part of the liquid helium container are housed two concentric copper disks acting as thermal shields and connected to the sample holder support with two Rexilon rods (10 $\mathrm{mm}$ diameter), useful to minimize the thermal conduction between the immersed components and the thermal shields.

The sample holder support consists of a PEEK holder support housing two superconducting NbTi coils attached with concentric tubes with $21 \mathrm{~mm}$ apertures pointing to the sample set in the center. Considering the aperture dimension of the magnetic coils, the maximum sample size can be $20 \times 20 \mathrm{~mm}$ and can be replaced by simply opening the cryostat top flange [top dark part of Fig. 1(b)] since the sample holder and the magnetic coils are connected to the copper disks shields and connected to this flange. The coils generate

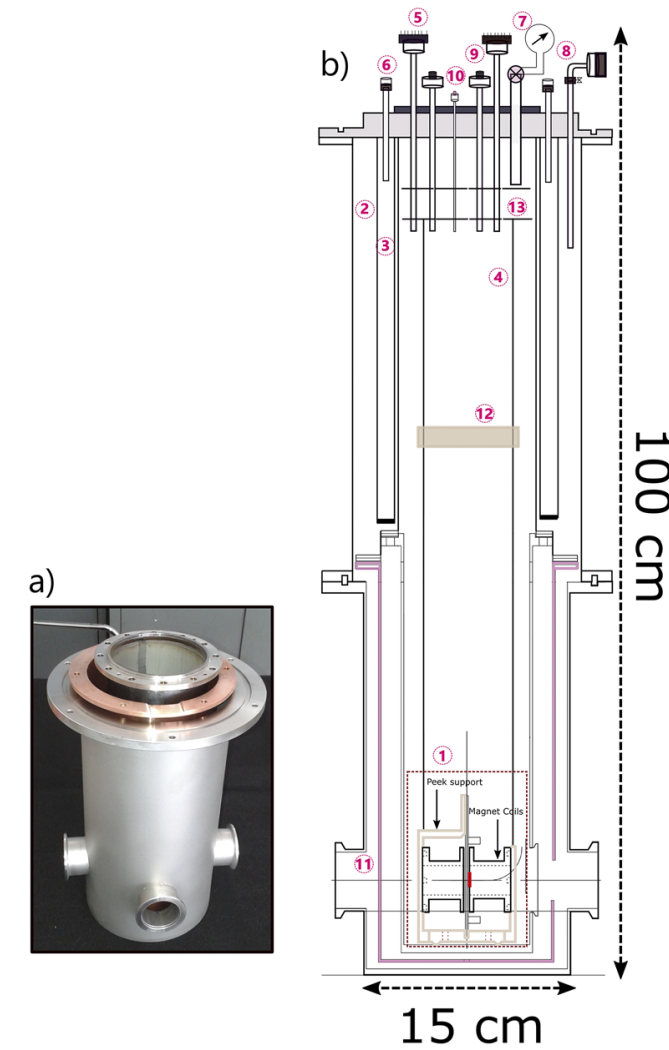

FIG. 1. (a) Image of the lower half of the cryostat showing the vacuum jacket, the copper shield, and the inner LHe container and (b) section of the optical cryostat. (1) Sample compartment inside the opto-magnetic setup; (2) the vacuum jacket; (3) the liquid nitrogen reservoir; (4) the liquid helium reservoir; (5) connector for signal, thermometer, and gauss-meter wiring; (6) liquid nitrogen insert tube; (7) liquid helium pressurizing and pumping valve; (8) vacuum jacket pumping valve; (9) liquid helium insert tube; (10) LHe level sensor feedthrough; (11) copper radiation shield; (12) Teflon support of the Rexilon rods; and (13) two copper disks as radiation shields. The sample holder is connected to the lower disk via two Rexilon rods to minimize thermal losses.

a magnetic field normal to the sample surface plane (see Fig. 2) up to $150 \mathrm{mT}$ at $4.2 \mathrm{~K}$ calibrated with a calibrated gaussmeter. Under operation, the vacuum jacket chamber reaches up to $1 \times 10^{-4} \mathrm{mbar}$ rapidly, while the vacuum level lowers to $5 \times 10^{-6}$ mbar, introducing liquid nitrogen inside the LN bath. The latter should be refilled periodically prior to introduction of liquid helium. The thermal conduction from the bottom and the contact thermal conduction lead to a temperature of $\sim 100 \mathrm{~K}$ in the main cylinder upon insertion of liquid nitrogen. The LHe insertion takes place in an over pressurized He inner cryotube to prevent crystallization on the sample surface. The temperature is read by using a calibrated Cernox thermometer connected to the PCB sample support, and the applied magnetic field is measured via a previously calibrated Hall probe inserted perpendicularly inside the coil at the back of the sample (see Fig. 3). The magnetic field can be modulated via the applied current to the superconducting coil with a resolution of $1 \mathrm{mT}$ (for a current supply of $10 \mathrm{~mA}$ ). The cryostat has the capacity of $\sim 5 \mathrm{Lt}$, which reaches the minimum temperature of $4.2 \mathrm{~K}$ within $40 \mathrm{~min}$ 


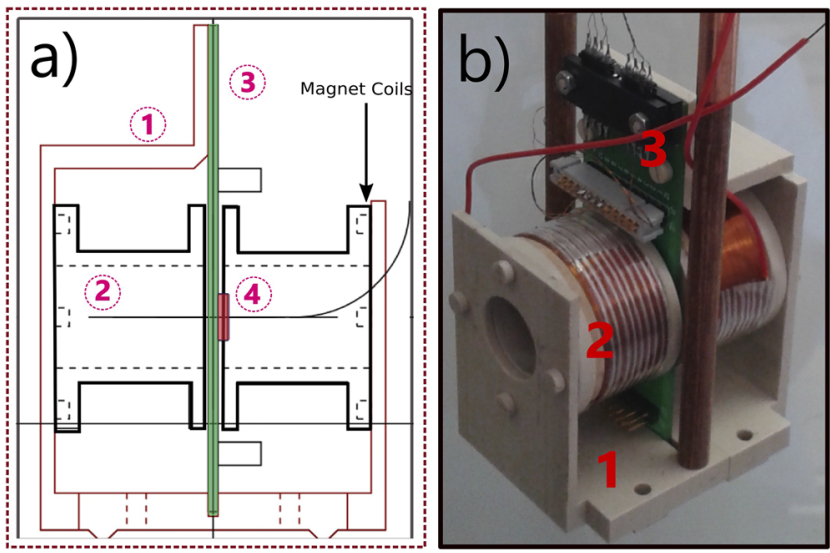

FIG. 2. (a) The layout of the sample holder: (1) the PEEK sample holder, (2) the superconducting magnetic coils made by NbTi wires, (3) PCB sample support and electrical contacts, and (4) the sample placed in the center. (b) Image of the sample holder.

upon insertion of LHe, depending on the LHe flux. The temperature is stable within $\Delta=0.05 \mathrm{~K}$ and $\Delta=0.1 \mathrm{~K}$ without and with magnetic fields, respectively (see Fig. 3). The cryostat maintains the temperature $4.2 \mathrm{~K}$ for about $6 \mathrm{~h}$ without incident radiations and in the presence of the applied magnetic field. The LN evaporator jacket reduces bubbling inside the cryotube, a condition that significantly reduces the light scattering along the optical path. ${ }^{4}$ The temperature can be further reduced down to $\sim 2 \mathrm{~K}$ with a continuous pumping of the pressurized LHe bath. The input/output signal cables are shielded via EMI capacitor filters (EMIFIL-NFE61PT), enclosed in a metallic box to further reduce the environmental noise and connected to a high insulating Fischer connector. A test on a niobium device with a dynamic resistance of $1 \Omega$ and a $100 \mu \mathrm{A}$ supply current revealed a noise of $0.0089 \mu \mathrm{V} / \sqrt{\mathrm{Hz}}$ with the standard deviation of $\sigma=0.00085 \mu \mathrm{V}$ at $4.2 \mathrm{~K}$.
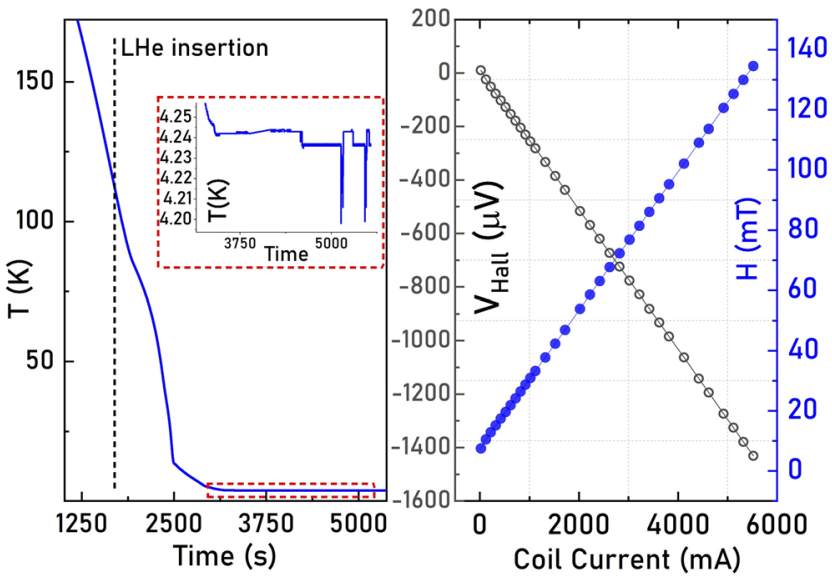

FIG. 3. (Left) The cooling behavior of the cryostat upon LHe insertion vs time. The temperature stability at $4.2 \mathrm{~K}$ (Inset). (Right) The Hall voltage measurement and its corresponding magnetic field generated via the magnetic coils at $4.2 \mathrm{~K}$.
The detailed cryogenic optical arrangement is described in Fig. 4. The layout consists of a $2 \mathrm{~mm}$ Zeonex window mounted with a Viton O-ring to the vacuum chamber jacket and connected to the ambient temperature. Zeonex is a cyclo-olefin material that exhibits the highest transmission at long wavelengths, while it does not show a noticeable dispersion in this frequency range. Along the optical path, the second window is in contact with LHe under a heavy tensile pressure (due to the differential pressure between the two volumes). The window is made by $2.2 \mathrm{~mm}$ thick $\mathrm{z}$-cut crystal quartz as will be discussed later. The window is connected to the LHe cryotube by a modified Conflat flange, and the vacuum is sealed by a Teflon ring on the vacuum side and an indium wire on the liquid side (see inset 1 of Fig. 4). The window was tightened in gradual cross contraction using a torque driver up to $2 \mathrm{~N} / \mathrm{m}$.

A $2 \mathrm{~mm}$ diameter Zeonex focusing lens with a $20 \mathrm{~mm}$ focal distance is set in from the sample, screwed within the coil aperture inside the PEEK cylinder support (see inset 2 of Fig. 4). The cylinder itself is fixed by a PEEK nut tightening the cylinder in place. This design further facilitates the setting along the axis of the lens focal point with regard to the sample positioning. The optimal thickness of all windows, being the minimum thickness capable of withstanding the pressure difference insuring its high transmittance, was calculated considering the material tensile strengths as

$$
d=\sqrt{\frac{1.1 \times P \times R^{2} \times S}{\eta}},
$$

where $d$ is the slab thickness, $R$ is the unsupported area, $P$ is the pressure difference, $S$ is the safety factor, and $\eta$ is the modulus of the rupture. The relative thickness behavior vs differential pressure of the crystal quartz with $\eta=9427$ psi with a maximum diameter of $30 \mathrm{~mm}$ unsupported area is shown in Fig. 5. Considering the differential pressure to sustain, a minimum thickness in the range $d=1.8 \mathrm{~mm}-2.2 \mathrm{~mm}$ was considered. While for the outer Zeonex window with $\eta=6090$ psi withstanding the atmospheric pressure, a relatively smaller thickness of the windows has been considered $(2 \mathrm{~mm})$. Both Zeonex and crystal quartz windows were tested under these conditions and passed the tests. The Zeonex window was also tested under vacuum down to $1 \times 10^{-6}$ mbar.

In order to evaluate the effective transmission and the efficiency of the optical layout, the optical response of the Zeonex windows was measured in the spectral region of interest. The optical response of the polymeric Zeonex material was investigated with terahertz time-domain spectroscopy (THz-TDS), described in detail in Refs. 26-28. The in-house THz-TDS spectrometer in the transmission configuration, used for this purpose, is based on two photoconductive switches: one as the emitter of the broadband THZ radiation $(0.1 \mathrm{THz}-2.5 \mathrm{THz})$ and one as the receiver, ${ }^{29}$ respectively [see Fig. 6(a)]. Two twin G10620-11 Hamamatsu photo-conductive antennas (PCAs) were operated by using a mode-locked ultrafast laser (FemtoFiber NIRpro, Toptica), at $780 \mathrm{~nm}$, a temporal pulse width of $100 \mathrm{fs}$, a repetition rate of $80 \mathrm{MHz}$, and an output power of $150 \mathrm{~mW}$. After the laser power reduction, a beam splitter (BS) 50:50 splits the main beam into two separated pump and probe beams. Dielectric mirrors propagate the beams toward emitter and receiver PCAs. The $\mathrm{THz}$ produced by the emitter is then collected and collimated by TPX lenses with a focal length of $50 \mathrm{~mm}$. The 

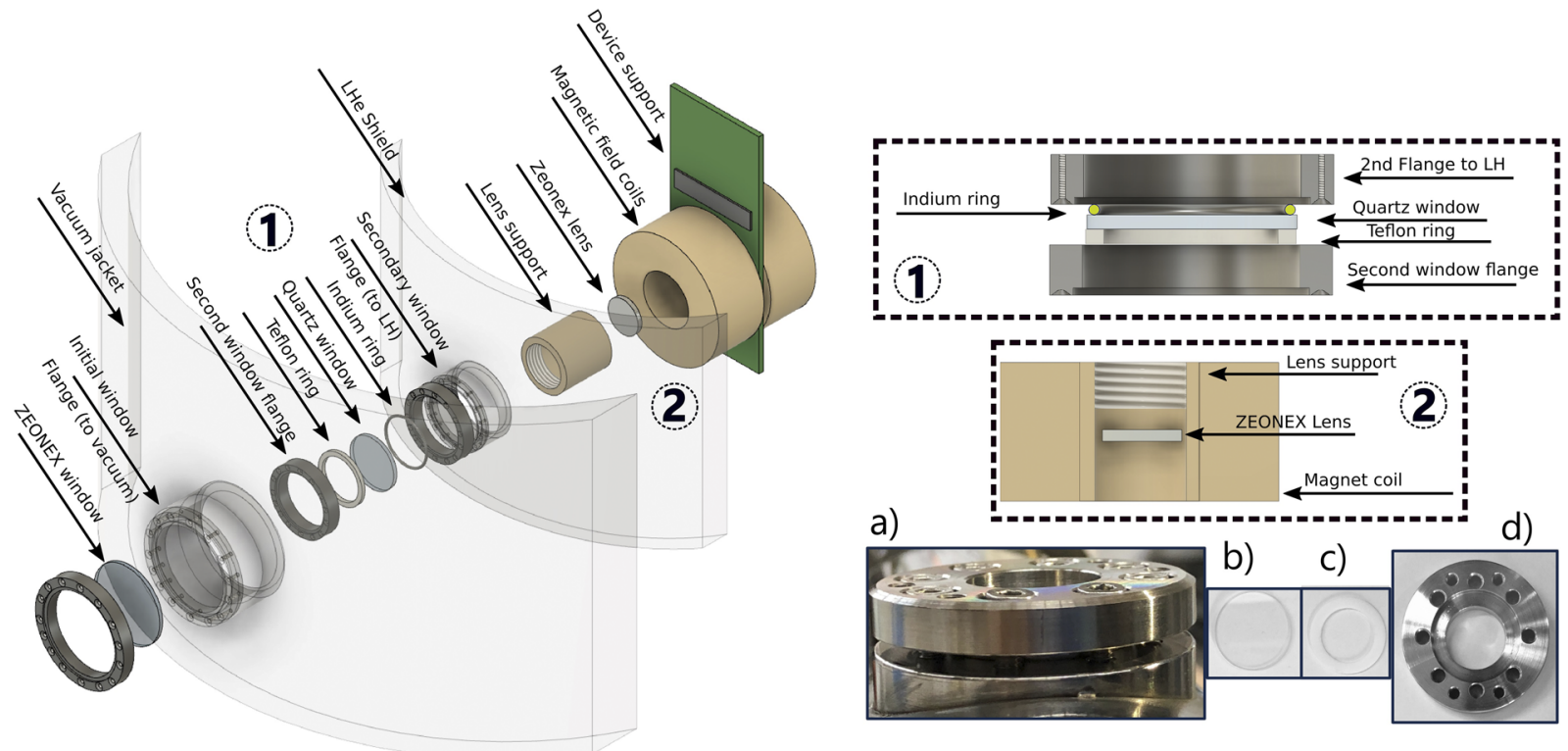

a)

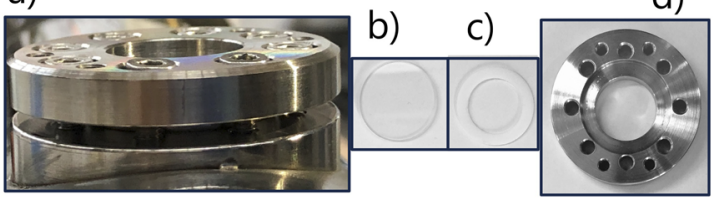

FIG. 4. Layout of the optical setup from the outer vacuum jacket of the cryostat to the inner coil lens positioning. The two segments indicated by numbers 1 and 2 are shown disassembled and in the insets after the assembly. (a) The actual configuration as indicated in frame (1); (b) the optical lens used in the layout; (c) the Teflon ring; and (d) the modified Conflat flange.

$\mathrm{THz}$ beam is focused on the sample, and the transmitted radiation is recollected, re-collimated, and refocused on the receiver PCA. Simultaneously, the probe beam is used to gate the $\mathrm{THz}$ detector PCA. Two motorized stages are used in the system configuration (see Fig. 6). The first one is a delay line (DDSM100/M, Thorlabs) that allows the collection of the temporal $\mathrm{THz}$ electric field, varying the optical path between pump and probe beams. ${ }^{26}$ The second one is a three-axis motor stage that ensures an accurate positioning and alignment of the sample in the $\mathrm{THz}$ beam focal spot.

The output signal from the $\mathrm{THz}$ detector, filtered by using a lock-in amplifier, was acquired and digitized and then transferred

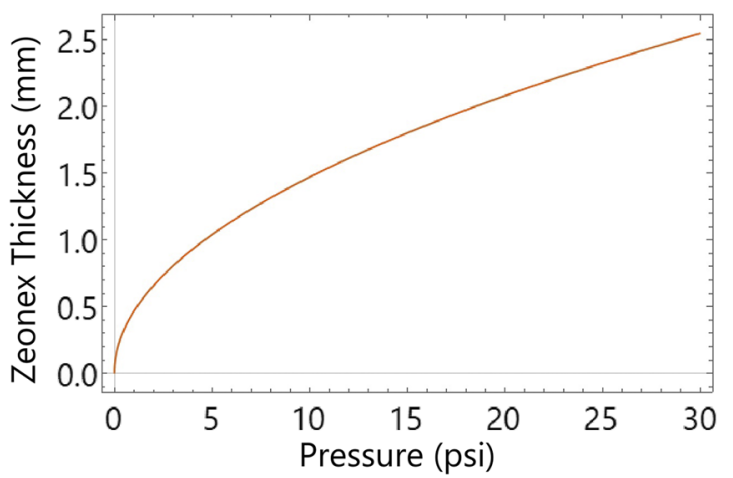

FIG. 5. The curve of the Zeonex window slab thickness as a function of the pressure difference between the cryostat jackets. to the PC. The optical system provides a spectral bandwidth ranging from $0.1 \mathrm{THz}$ to $2.5 \mathrm{THz}$, with a spectral resolution of $50 \mathrm{GHz}$. This spectral resolution is sufficient to characterize the response of the non-resonant and transparent window set. We recorded $5 \mathrm{THz}$ electric field profiles with five scans. The $\mathrm{THz}$ electric fields for the free space (without sample) and for the Zeonex window with thickness $2 \mathrm{~mm}$ are shown in Fig. 6(b). The temporal delay between the air and the Zeonex signal was $3.75 \pm 0.01$ ps. The power spectra of free space and Zeonex slab are also shown in Fig. 6(c) along with its total transmittance [see Fig. 6(d)]. The transmittance was calculated as

$$
T_{\text {Zeonex }}=\frac{\left|E_{\text {Zeonex }}(\omega)\right|^{2}}{\left|E_{\text {Freespace }}(\omega)\right|^{2}} .
$$

The results indicate a transmission of about $80 \%$ in the range below $1 \mathrm{THz}$, which is slightly higher than that of crystal quartz.

The total transmission of the optical arrangement was then calculated using the Beer-Lambert law ${ }^{31}$ and considering the transmission of the Zeonex derived from experimental results (see Fig. 7). In order to prevent any contamination of visible and IR radiation inside the cryotube, a $0.2 \mathrm{~mm}$ flexible HDPE filter (with $\mathrm{T} \sim 85 \%$ at $1 \mathrm{THz}^{32}$ ) was attached to the entrance window. Based on the thickness of the Zeonex windows, crystal quartz, and the filter transmission, a total transmission of about $45 \%-50 \%$ onto the sample was calculated.

Based on the geometry of the cryogenic system, three different layouts of the optical paths were considered, as described in Fig. 8. All of them are possible configurations of this optical system. Key parameters, considered mainly in the lens design, were the resolution, the focal length, and the lens diameter. Both focal length and 
a)

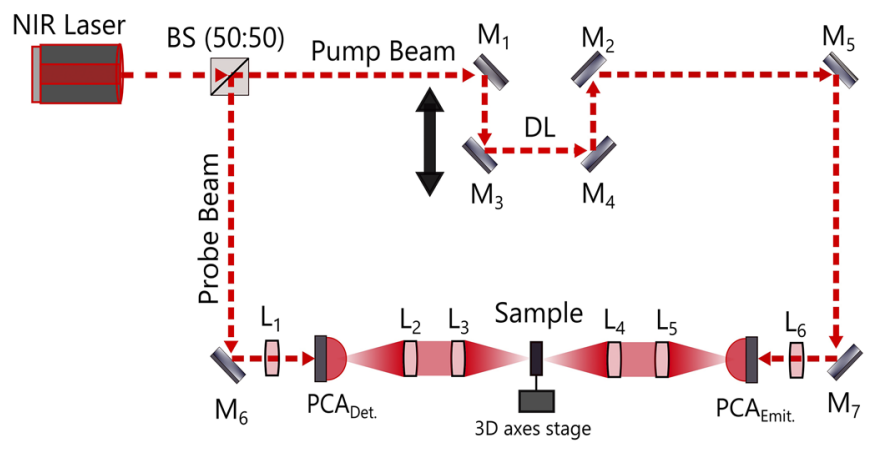

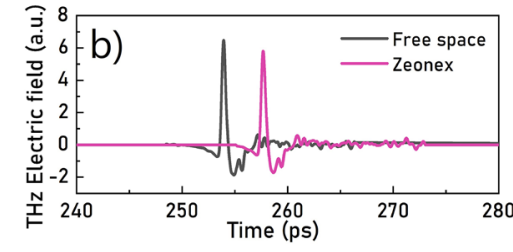
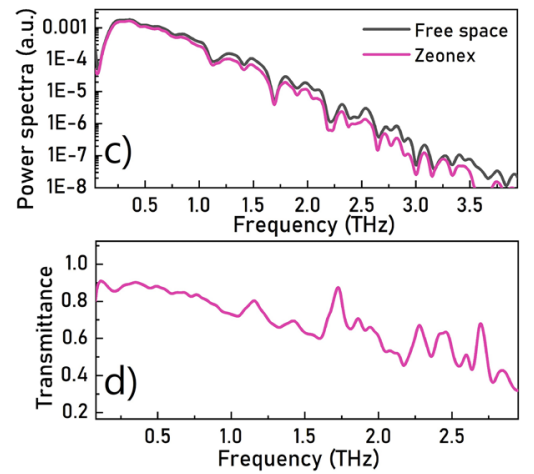

FIG. 6. (a) THz-TDS system in transmission geometry for spectroscopic characterization of the Zeonex window. (b) THz electric fields: THz electric field relative to free space (black line) and THz transmitted electric field of the Zeonex window (red line); (c) power spectra relative to free space (black line) and 2 mm thick Zeonex window (red line); (d) transmittance of $2 \mathrm{~mm}$ thick Zeonex window slab.

lens diameter are determined by geometrical constraints. Furthermore, we planned to have a focal spot diameter less than $1 \mathrm{~mm}$ in order to guarantee a sufficient number of photons on the sample. In layout (1), a $\mathrm{THz}$ plane wave illuminates the focusing lens (L1) with a focal length of $130 \mathrm{~mm}$. A polymeric/quartz window (W1) is located between the lens (L1) and its focal plane. The maximum diameter for the lens in this location is $50 \mathrm{~mm}$. In layout (2), the $\mathrm{THz}$ radiation passes the polymeric window (W1), and it is focused by the lens (L1) with a focal length of about $50 \mathrm{~mm}$ that also acts as the second window. In layout (3), the plane wave is incident on two polymeric/quartz windows (W1) and (W2), and it is finally focused

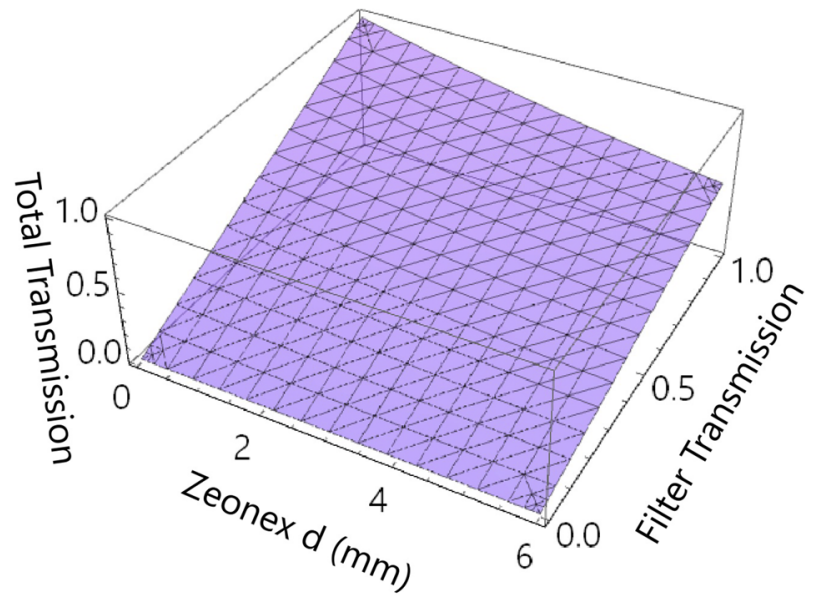

FIG. 7. The total transmission of the optical layout with the Zeonex windows and the IR-Vis filter. by the lens (L1) with a focal length of about $20 \mathrm{~mm}$ (length of the magnetic coil aperture $\mathrm{L}=32 \mathrm{~mm}$ ). Considering the constraints of the coil, the maximum lens diameter is $20 \mathrm{~mm}$ since the diameter of the magnetic coil aperture is $\mathrm{D}=21 \mathrm{~mm}$.

All layouts have been numerically simulated by means of a commercial software based on the finite element method in the frequency domain. ${ }^{33}$ The material selected for both windows and lenses is the Zeonex by Zeon Corporation, a cyclo-olefin with a refractive index of $\epsilon=1.518-j 0.001$ at $1 \mathrm{THz}$, successfully employed in other devices operating at $\mathrm{THz}$ frequencies. ${ }^{34,35}$ The windows have been designed with a thickness of $2 \mathrm{~mm}$. The lens (L1) in layout 1 is a plano-convex lens with a radius of curvature $\mathrm{R}=67.6 \mathrm{~mm}$ and a maximum thickness of $1 \mathrm{~cm}$. The lens (L1) in layout (3) is a planoconvex lens with a radius of curvature $\mathrm{R}=10.7 \mathrm{~mm}$ and a maximum thickness of $1 \mathrm{~cm}$. The lens (L1) in layout (2) is a plano-convex lens with a radius of curvature $\mathrm{R}=26.2 \mathrm{~mm}$ and a maximum thickness of $1 \mathrm{~cm}$. As a proof of principle, a linearly polarized plane wave has been selected as a radiation source at $1 \mathrm{THz}$.

The results of the numerical simulations are described in Fig. 8 . They confirm the occurrence of a focal spot with a diameter comparable with the theory. ${ }^{31}$ The full width at half maximum (FWHM) of the absolute value of the electric field in the focal area corresponds to a measure of the focal spot diameter of the selected lenses. The FWHM of the lens $\left(\mathrm{L}_{1}\right)$ focal spot is equal to about $1 \mathrm{~mm}$ for layout (1), $0.48 \mathrm{~mm}$ for layout (3), and $0.65 \mathrm{~mm}$ for layout (2). As expected, the presence of the windows slightly affects the focusing properties in terms of maximum values of the electric field modulus at the focal point due to the mismatch of the refractive index between vacuum and Zeonor and to the moderate absorption of the polymer at $1 \mathrm{THz}$. The optical configuration presented in layout (3) shows a higher resolution of the lens $\left(\mathrm{L}_{1}\right)$ at $1 \mathrm{THz}$, compared with the other configurations. Hence, we selected the latter optical arrangement for the 


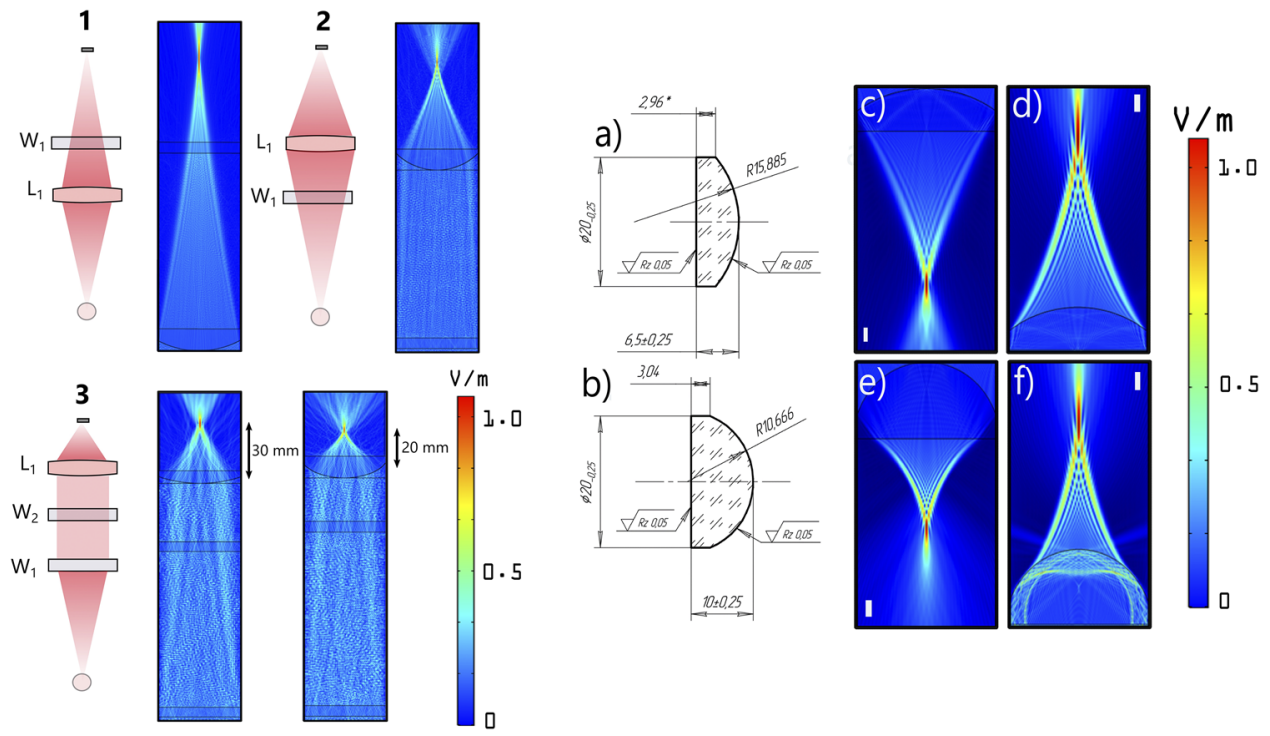

FIG. 8. [(1)-(3)] Different optical configurations based on the geometry of the cryostat and their simulations; (1) a single outer jacket lens and a single inner window; (2) a single outer jacket window and a single inner tube lens; (3) an outer jacket window, an inner tube window, and one very close lens; (a)-(f) different lens configurations and their DoF calculation. The bars show the normalized modulus of the electric field. cryogenic system that was designed and manufactured ${ }^{32}$ according to layout (3).

Furthermore, to implement the tuning of the lens depth of field (DoF), defined as the distance along the optical axis in which the power does not decrease below $80 \%$ of the maximum, a second lens with a focal length of $30 \mathrm{~mm}$ alternative to the lens (L1) of layout (3) was numerically simulated. The modulation of the DoF facilitates the compensation of the photon intensity attenuation due to sample positioning misalignments. The plano-convex lens with a focal length of $30 \mathrm{~mm}$ has a diameter of $20 \mathrm{~mm}$, a radius of curvature $\mathrm{R}$ $=15.9 \mathrm{~mm}$, and a maximum thickness of $6.5 \mathrm{~mm}$. Simulations of this lens in layout (3) are reported in Fig. 8. The FWHM of the resulting focal spot is equal to about $0.56 \mathrm{~mm}$. Simulations confirm that a longer focal length allows a longer DoF. We also evaluated the lens orientation in term of DoF by numerical simulations. As reported in Fig. 8, when the lens is oriented as in Figs. 8(c) and 8(e), DoF is $2.3 \mathrm{~mm}$ and $3.5 \mathrm{~mm}$ for the lens with a focal length of $20 \mathrm{~mm}$ and $30 \mathrm{~mm}$, respectively. On the other hand, when the lens is oriented as in Figs. 8(d) and 8(f), DoF is $3.5 \mathrm{~mm}$ and $4.2 \mathrm{~mm}$ for the lens with a focal length of $20 \mathrm{~mm}$ and $30 \mathrm{~mm}$, respectively. The simulations confirm that the selected lenses guarantee the necessary flexibility with respect to the sensor position and with acceptable losses. Furthermore, Zeonex does not show a noticeable dispersion and has a refractive index deviation of about 0.002 in the frequency range between $200 \mathrm{GHz}$ and $4.2 \mathrm{THz}{ }^{36}$ Hence, this difference does not affect the focusing properties of the lenses.

Finally, the total transmission and efficiency of the optical configuration was measured at the optical exit using a continuous diode $\mathrm{THz}$ emitter at $270 \mathrm{GHz}$ (Virginia Diode).

The total transmission of the optical line was measured using a calibrated pyroelectric detector with the voltage sensitivity of $\eta$ $=8000 \mathrm{~V} / \mathrm{W}$, positioned at the sample position in the center of the magnetic coil aperture. The incident beam was chopped at $16 \mathrm{~Hz}$ and sent through the optical line of the cryostat [see Fig. 9(d)]. The pyroelectric response was triggered and measured with and without the optical line, moving the final stage of the focusing lens. The lens was shifted in a $5 \mathrm{~mm}$ range to optimize the focal distance and the transmission. The measurement was performed looking at the
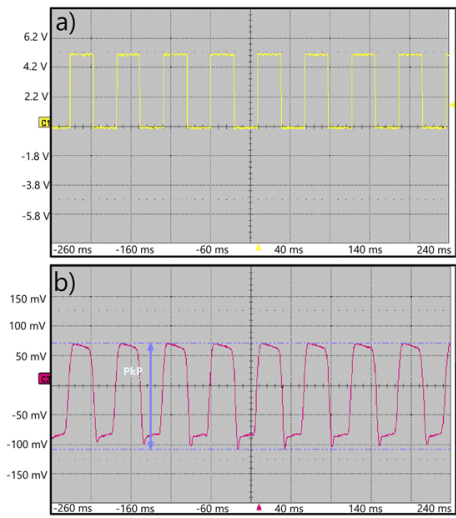

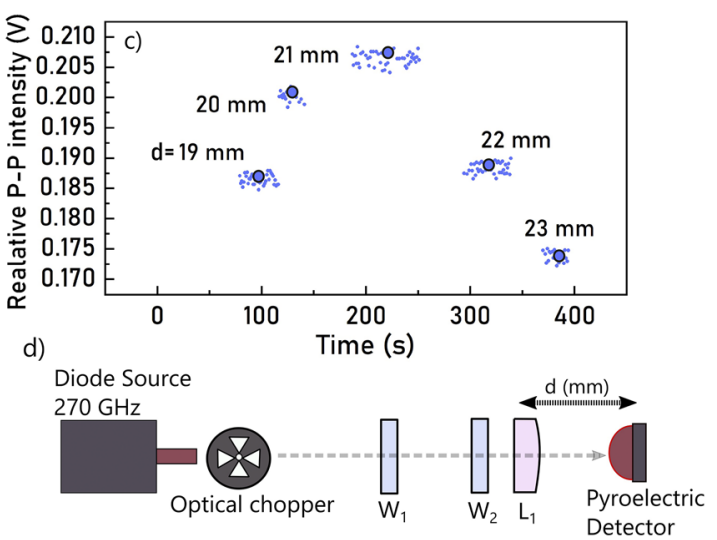

FIG. 9. The overall transmission measurements on the optical path and the optimization of the lens focus on the sample positioning; (a) oscilloscope screenshot of the chopper trigger signal; (b) screenshot of the photo-response of the diode; (c) the measured photoresponse values from the peak to peak measurement of the amplified potential $\left(V_{p-p}\right)$ of the pyroelectric sensor; (d) the measurement setup with the optical line with the two windows and the $20 \mathrm{~mm}$ focal length lens. 
peak to peak amplified voltage response of the pyroelectric detector triggered by the output frequency of the chopper [see Figs. 9(a) and 9(b)].

The lens distance was varied via the perforated support designed for an accurate translation inside the magnetic coil aperture. The lens with the focal distance of $20 \mathrm{~mm}$ was used in these tests, and the distance changed from $\mathrm{d}=19$ to $\mathrm{d}=23 \mathrm{~mm}$ using the mounted screw. As can be seen in Fig. 9(c), the lens shows a relatively asymmetric response, while the highest intensity was measured at $f$ $=21 \mathrm{~mm}$ for the value of $\mathrm{V}_{p-p}=210 \mathrm{mV}$. The deviation from the maximum in the $4 \mathrm{~mm}-5 \mathrm{~mm}$ distance range was measured to be of the order of $\Delta I_{d}= \pm 24 \%$, ranging from $\mathrm{V}_{p-p}=175 \mathrm{mV}$ to $\mathrm{V}_{p-p}$ $=210 \mathrm{mV}$. The result is in agreement with the simulation of the optical response of the lens. The depth of focus determined allows measurements with minor displacements of the sample positioning. The overall transmission of the optical line in the cryotube without LHe was also calculated to be $42 \%$ in relatively good agreement with the estimated value of $\sim 50 \%$, slightly lower due to the transmission difference at these wavelengths. The cryogenic optical setup shows an acceptable response and hence could be successfully used for the investigation of new proximity array devices that require a sufficient number of photon and the control over external magnetic and electric fields. ${ }^{22,}$

\section{CONCLUSIONS}

We presented here the characteristics of a compact liquid helium immersion cryostat designed and built with an optical setup optimized for experiments using radiation at long wavelengths. The system has been specifically designed to characterize novel proximity junction array detectors and other 2D imaging detectors working at $\mathrm{LHe}$ temperature. The cryostat allows us to maintain the sample at the temperature of $4.2 \mathrm{~K}$ for $6 \mathrm{~h}$, while it may reach the lowest temperature of $2 \mathrm{~K}$ upon LHe bath pumping. A liquid nitrogen boiling chamber is used to reduce bubbling inside the cryotube. The optical configuration of the device is made by three stages of optical elements: a Zeonex window, a crystal quartz window, and a Zeonex focusing lens out of which the latter two are immersed in liquid helium. All materials of the optical windows were selected on the basis of accurate simulations and have a thickness of about $2 \mathrm{~mm}$, i.e., $2 \mathrm{~mm}$ for Zeonex and $2.2 \mathrm{~mm}$ for crystal quartz, considering both material tensile stress and the differential pressure they are withstanding inside the cryostat and their optical parameters. The transmission of the Zeonex windows was measured using two stage photo-diode configurations, and for a thickness of $2 \mathrm{~mm}$, they are characterized by a transmission of $80 \%$ at $1 \mathrm{THz}$. Based on the optical layout, different configurations were simulated with a spatial resolution ranging from $0.48 \mathrm{~mm}$ to $1 \mathrm{~mm}$, and the configuration with the highest estimated resolution was realized. Furthermore, several other configurations were simulated and compared to verify the tunability of the DoF in the range $2.3 \mathrm{~mm}-8 \mathrm{~mm}$ in order to compensate sample positioning misalignments. The total transmission of the optical configuration exhibits a maximum value of $42 \%$, while a DoF deviation of $24 \%$ was measured by changing the lens focal distance. These results measured in cryogenic conditions are in good agreement with theoretical estimations. Summarizing, this original cryogenic system matches the demanding requests of the investigation of samples and devices to be probed at low temperature, long wavelengths, and when external electric and magnetic fields have to be applied.

\section{ACKNOWLEDGMENTS}

This project has been supported within the framework of the TERA project of the fifth Committee of the INFN. We would like to thank S. Cibella and P. Carelli for their support in the assembly of the apparatus for pyroelectric measurements and A. Grilli and A. Raco for their valuable support in the construction of the apparatus. We acknowledge the financial support from the Bilateral Cooperation Agreement between Italy and Japan of the Italian Ministry of Foreign Affairs and the International Cooperation (MAECI) in the framework of the project of major relevance N. PGR0072.

\section{DATA AVAILABILITY}

The data that support the findings of this study are available within the article.

\section{REFERENCES}

${ }^{1}$ M. Niedermayr, K. Lakhmanskiy, M. Kumph, S. Partel, J. Edlinger, M. Brownnutt, and R. Blatt, "Cryogenic surface ion trap based on intrinsic silicon," New J. Phys. 16(11), 113068 (2014).

${ }^{2}$ M. Schwarz, O. O. Versolato, A. Windberger, F. R. Brunner, T. Ballance, S. N. Eberle, J. Ullrich, P. O. Schmidt, A. K. Hansen, A. D. Gingell, M. Drewsen, and J. R. C. López-Urrutia, "Cryogenic linear Paul trap for cold highly charged ion experiments,” Rev. Sci. Instrum. 83(8), 083115 (2012).

${ }^{3}$ P. Micke, J. Stark, S. A. King, T. Leopold, T. Pfeifer, L. Schmöger, M. Schwarz, L. J. Spieß, P. O. Schmidt, and J. R. Crespo López-Urrutia, "Closed-cycle, low-vibration 4 k cryostat for ion traps and other applications,” Rev. Sci. Instrum. 90(6), 065104 (2019).

${ }^{4}$ S. S. Andrews and S. G. Boxer, "A liquid nitrogen immersion cryostat for optical measurements,” Rev. Sci. Instrum. 71(9), 3567-3569 (2000).

${ }^{5}$ S. S. Dhillon, M. S. Vitiello, E. H. Linfield, A. G. Davies, M. C. Hoffmann, J. Booske, C. Paoloni, M. Gensch, P. Weightman, G. P. Williams, E. Castro-Camus, D. R. S. Cumming, F. Simoens, I. Escorcia-Carranza, J. Grant, S. Lucyszyn, M. Kuwata-Gonokami, K. Konishi, M. Koch, C. A. Schmuttenmaer, T. L. Cocker, R. Huber, A. G. Markelz, Z. D. Taylor, V. P. Wallace, J. A. Zeitler, J. Sibik, T. M. Korter, B. Ellison, S. Rea, P. Goldsmith, K. B. Cooper, R. Appleby, D. Pardo, P. G. Huggard, V. Krozer, H. Shams, M. Fice, C. Renaud, A. Seeds, A. Stohr, M. Naftaly, N. Ridler, R. Clarke, J. E. Cunningham, and M. B. Johnston, "The 2017 terahertz science and technology roadmap," J. Phys. D: Appl. Phys. 50(4), 043001 (2017).

${ }^{6}$ M. Ortolani, S. Lupi, L. Baldassarre, U. Schade, P. Calvani, Y. Takano, M. Nagao, T. Takenouchi, and H. Kawarada, "Low-energy electrodynamics of superconducting diamond,” Phys. Rev. Lett. 97(9), 097002 (2006).

${ }^{7}$ E. A. Nanni, W. R. Huang, K.-H. Hong, K. Ravi, F. Arya, G. Moriena, R. J. Dwayne Miller, and F. X. Kärtner, "Terahertz-driven linear electron acceleration," Nat. Commun. 6(1), 8486 (2015).

${ }^{8}$ P. U. Jepsen, D. G. Cooke, and M. Koch, “Terahertz spectroscopy and imagingModern techniques and applications," Laser Photonics Rev. 5(1), 124-166 (2011).

${ }^{9}$ D. Saeedkia, Handbook of Terahertz Technology for Imaging, Sensing and Communications (Woodhead Publishing Limited, 2013).

${ }^{10}$ B. S. Karasik, A. V. Sergeev, and D. E. Prober, "Nanobolometers for THz photon detection," IEEE Trans. Terahertz Sci. Technol. 1(1), 97-111 (2011).

${ }^{11}$ A. Toma, S. Tuccio, M. Prato, F. De Donato, A. Perucchi, P. Di Pietro, S. Marras, C. Liberale, R. Proietti Zaccaria, F. De Angelis, L. Manna, S. Lupi, E. Di Fabrizio, and L. Razzari, "Squeezing terahertz light into nanovolumes: Nanoantenna enhanced terahertz spectroscopy (NETS) of semiconductor quantum dots," Nano Lett. 15(1), 386-391 (2015). 
${ }^{12}$ F. D’Apuzzo, A. R. Piacenti, F. Giorgianni, M. Autore, M. Cestelli Guidi, A. Marcelli, U. Schade, Y. Ito, M. Chen, and S. Lupi, "Terahertz and midinfrared plasmons in three-dimensional nanoporous graphene," Nat. Commun. 8(1), 14885 (2017).

${ }^{13}$ S. J. Rezvani, N. Pinto, L. Boarino, F. Celegato, L. Favre, and I. Berbezier, "Diffusion induced effects on geometry of Ge nanowires," Nanoscale 6, 7469-7473 (2014).

${ }^{14}$ S. J. Rezvani, N. Pinto, E. Enrico, L. D’Ortenzi, A. Chiodoni, and L. Boarino, "Thermally activated tunneling in porous silicon nanowires with embedded Si quantum dots," J. Phys. D: Appl. Phys. 49(10), 105104 (2016).

${ }^{15}$ N. Pinto, S. J. Rezvani, L. Favre, I. Berbezier, M. Fretto, and L. Boarino, "Geometrically induced electron-electron interaction in semiconductor nanowires," Appl. Phys. Lett. 109(12), 123101 (2016).

${ }^{16}$ N. Pinto, S. J. Rezvani, A. Perali, L. Flammia, M. V. Milošević, M. Fretto, C. Cassiago, and N. De Leo, "Dimensional crossover and incipient quantum size effects in superconducting niobium nanofilms," Sci. Rep. 8(1), 4710 (2018).

${ }^{17}$ S. Carturan, G. Maggioni, S. J. Rezvani, R. Gunnella, N. Pinto, M. Gelain, and D. R. Napoli, "Wet chemical treatments of high purity Ge crystals for $\gamma$-ray detectors: Surface structure, passivation capabilities and air stability," Mater. Chem. Phys. 161, 116-122 (2015).

${ }^{18}$ S. D. Sartale, A. A. Ansari, and S.-J. Rezvani, "Influence of Ti film thickness and oxidation temperature on $\mathrm{TiO}_{2}$ thin film formation via thermal oxidation of sputtered Ti film," Mater. Sci. Semicond. Process. 16(6), 2005-2012 (2013).

${ }^{19}$ S. J. Rezvani, R. Gunnella, D. Neilson, L. Boarino, L. Croin, G. Aprile, M. Fretto, P. Rizzi, D. Antonioli, and N. Pinto, "Effect of carrier tunneling on the structure of Si nanowires fabricated by metal assisted etching," Nanotechnology 27(34), 345301 (2016).

${ }^{20}$ S. J. Rezvani, M. Pasqualini, A. Witkowska, R. Gunnella, A. Birrozzi, M. Minicucci, H. Rajantie, M. Copley, F. Nobili, and A. Di Cicco, "Binder-induced surface structure evolution effects on Li-ion battery performance," Appl. Surf. Sci. 435, 1029-1036 (2018).

${ }^{21}$ M. Pasqualini, S. Calcaterra, F. Maroni, S. J. Rezvani, A. Di Cicco, S. Alexander, H. Rajantie, R. Tossici, and F. Nobili, "Electrochemical and spectroscopic characterization of an alumina-coated $\mathrm{LiMn}_{2} \mathrm{O}_{4}$ cathode with enhanced interfacial stability," Electrochim. Acta 258, 175-181 (2017).

${ }^{22}$ J. Rezvani, D. Di Gioacchino, C. Gatti, N. Poccia, C. Ligi, S. Tocci, M. Cestelli Guidi, S. Cibella, S. Lupi, and A. Marcelli, "Tunable vortex dynamics in proximity junction arrays: A possible accurate and sensitive 2D THz detector," Acta Phys. Pol. A 137(1), 17-20 (2020).
${ }^{23}$ D. D. Gioacchino, N. Poccia, M. Lankhorst, C. Gatti, B. Buonomo, L. Foggetta, A. Marcelli, and H. Hilgenkamp, "A novel particle/photon detector based on a superconducting proximity array of nanodots," J. Supercond. Novel Magn. 30(2), 359-363 (2017)

${ }^{24}$ N. Poccia, T. I. Baturina, F. Coneri, C. G. Molenaar, X. R. Wang, G. Bianconi, A. Brinkman, H. Hilgenkamp, A. A. Golubov, and V. M. Vinokur, "Critical behavior at a dynamic vortex insulator-to-metal transition," Science 349(6253), 1202-1205 (2015).

${ }^{25}$ See http://dr.lnf.infn.it/technological-research/ for Tera project, $\operatorname{lnf}$, infn.

${ }^{26} \mathrm{E}$. Bianconi and M. Alfaro, "Photoconductive devices for terahertz pulsed spectroscopy: A review," Photonics Res. 4(3), A36-A42 (2016).

${ }^{27}$ B. Ferguson and X.-C. Zhang, "Materials for terahertz science and technology," Nat. Mater. 1(1), 26-33 (2002).

${ }^{28} \mathrm{X}$.-C. Zhang and J. Xu, Introduction to THz Wave Photonics (Springer US, Boston, MA, 2010).

${ }^{29}$ A. D’Arco, M. Di Fabrizio, V. Dolci, M. Petrarca, and S. Lupi, “THz pulsed imaging in biomedical applications," Condens. Matter 5, 25 (2020).

${ }^{30}$ H. J. Cha, Y. Uk. Jeong, S. Hee Park, B. Cheol Lee, and S. Han Park, "Transmission-property measurements of crystal quartz and plastic material in the terahertz region," J. Korean Phys. Soc. 49, 354-358 (2006).

${ }^{31}$ M. Born, E. Wolf, and E. Hecht, "Principles of optics: Electromagnetic theory of propagation, interference and diffraction of light," Phys. Today 53(10), 77-78 (2000).

${ }^{32}$ See http://www.tydexoptics.com for Tydex optics.

${ }^{33}$ See http://www.comsol.com for Comsol multiphysics.

${ }^{34}$ S. Tofani, D. C. Zografopoulos, M. Missori, R. Fastampa, and R. Beccherelli, "Terahertz focusing properties of polymeric zone plates characterized by a modified knife-edge technique,” J. Opt. Soc. Am. B 36(5), D88 (2019).

${ }^{35}$ S. Tofani, D. C. Zografopoulos, M. Missori, R. Fastampa, and R. Beccherelli, "High-resolution binary zone plate in double-sided configuration for terahertz radiation focusing," IEEE Photonics Technol. Lett. 31(2), 117-120 (2019).

${ }^{36}$ A. Podzorov and G. Gallot, "Low-loss polymers for terahertz applications," Appl. Opt. 47(18), 3254 (2008).

${ }^{37}$ S. J. Rezvani, D. D. Gioacchino, C. Gatti, C. Ligi, M. C. Guidi, S. Cibella, M. Fretto, N. Poccia, S. Lupi, and A. Marcelli, "Proximity array device: A novel photon detector working in long wavelengths," Condens. Matter 5(2), 33 (2020). 